\title{
Deutsch-Indische Joint Ventures in Indien
}

\author{
von Tillmann R. Braun
}

\section{A. Einführung ${ }^{1}$}

\section{Gegenstand der Untersuchung}

Indien ist mit $3287590 \mathrm{qkm}$ und 754 Millionen Einwohnern der Staat mit der zweithöchsten Bevölkerungszahl der Welt. ${ }^{2}$ Es tritt häufig als Sprecher der Entwicklungsländer auf. Mit einem Bruttosozialprodukt, das an fünfzehnter Stelle der Welt rangiert, ${ }^{3}$ bildet Indien durch das verhältnismäßig rasche Wachstum seiner Wirtschaft, seines Volkseinkommens und seiner Kaufkraft den größten homogenen Markt der Erde, der westlichen Industrienationen relativ offensteht. Die Erschließung dieses Binnenmarktes ist für die exportabhängige Industrie der Bundesrepublik Deutschland von großer Bedeutung.

Die vorliegende Untersuchung setzt sich mit einem Modell auseinander, das bei dieser Erschließung verwandt und als Gemeinschaftsunternehmen oder Joint Venture bezeichnet wird. Darunter wird die finanzielle, technische und führungsmäßige langfristige Zusammenarbeit zwischen indischen und deutschen Partnern verstanden, welche ihre gemeinschaftlichen Ziele in einer neuen selbständigen Rechtspersönlichkeit nach vertraglich geregelten Bedingungen verfolgen. ${ }^{4}$ Diese besonders enge Form der Kooperation gilt nach der angelsächsischen Terminologie als "Equity Joint Venture ". ${ }^{5}$ Sie stellt gleichsam einen Kompromiß zwischen den politischen Interessen der Entwicklungsländer an hinreichender Kontrolle und den wirtschaftlichen Zielen der Unternehmen aus den Industrienationen dar.

In dieser Untersuchung wird zunächst auf die Motive der deutschen und indischen Partner eingegangen, ein solches Gemeinschaftsunternehmen zu errichten. Nach einer kur-

1 Diese Untersuchung entstand im Rahmen eines Internships an der Deutsch-Indischen Industrie- und Handelskammer, welches der Autor in seinem Geburtsort Bombay im Frühjahr 1987 absolvierte.

2 Pendse, Statistical Outline of India 1986-87, Bombay 1986, S. 194.

3 Miller, India's Regulation of Foreign Direct Investment: Art. 29 of the 1973 FERA, in: Boston College Third World Law Journal 1981, S. 65 (67).

4 Vgl. die ausführliche empirische Studie von Becker, Thiel, Strukturen und Bewertungen deutsch-indischer Joint-Ventures in Indien. Ein Beitrag zur Entwicklungsforschung in: Joint Ventures in Asien, hrsg. von Karlheinz Hottes und Christian Uhlig, Bochum 1983, S. 36.

5 Im Gegensatz zum 'Contractual Joint Venture', welche alle weniger engen Kooperationsmöglichkeiten zusammenfaßt; dazu Zweigert/v. Hofmann, Zur internationalen .Joint Venture, in: FS-Martin Luther, München 1976, S. 203 (205). 
zen Darstellung der bestehenden deutsch-indischen Gemeinschaftsunternehmen erläutert sie wirtschaftliche und politische Gründe für die ambivalente Haltung Indiens gegenüber ausländischen Direktinvestitionen vor dem geschichtlichen Hintergrund der Entwicklung der indischen Wirtschaft (nachfolgend A. II -, IV). Daraufhin werden zwei für Deutsch-Indische Gemeinschaftsunternehmen wesentliche bilaterale völkerrechtliche Verträge, das Kapitalschutzabkommen und das Doppelsteuerungsabkommen, näher betrachtet (B.), da sie gleichsam einen völkerrechtlich verbindlichen Maßstab für die dann zu behandelnde innerstaatliche Investitionsgesetzgebung Indiens setzen (C.). Schließlich werden wesentliche Bestimmungen des indischen Gesellschaftsrechts und der vertraglichen Vereinbarungen Deutsch-Indischer Joint Ventures erörtert (D.).

\section{Deutsch-Indische Joint Ventures in Indien}

Von den in Betracht kommenden Strategien: Handel, Lizenzverträge, Gemeinschaftsunternehmen und vollbeherrschte Tochtergesellschaften stellen die internationalen Gemeinschaftsunternehmen nicht nur in Indien eine häufig anzutreffende Anlageform dar. Gegenüber den oft genannten Gründen für die Gründung eines Joint Ventures ${ }^{6}$ wie etwa Bearbeitung des asiatischen Marktes vom indischen Gemeinschaftsunternehmen aus oder Nutzung der kostengünstigen Produktionsweise spielt aus deutscher Sicht die Sicherung der durch Importrestriktionen gefährdeten Marktanteile bei weitem die wichtigste Rolle. Die Entscheidung für eine Produktionskooperation in Form eines Joint Ventures ist im wesentlichen eine Reaktion der deutschen Unternehmen auf die in Indien zum Schutz des indischen Binnenmarktes errichteten Importbarrieren. ${ }^{7}$ Diese nationalen Rahmenbedingungen stellten ausländische Unternehmen überhaupt vor die Alternative, entweder die schon erreichten Marktanteile in Indien zu verlieren und außerdem auf die Teilhabe am weiteren Wachstum zu verzichten oder aber mit Hilfe eines lokalen Partners in Indien zu produzieren und damit den indischen Markt direkt zu bedienen. Durch die Gründung von Gemeinschaftsunternehmen konnten nicht nur die Importrestriktionen umgangen, sondern auch die Möglichkeiten für weitere unternehmerische Aktivitäten in Indien offengehalten werden. Darüber hinaus ermöglichen sie durch "Einsatz von Kapital gegen Erwerb von Kontrolle ${ }^{8}$ noch Einflußnahme auf die Führung des Unternehmens.

Aus indischer Sicht wird die Entscheidung für ein Gemeinschaftsunternehmen mit dem Erwerb fortgeschrittener Technologie unter geringem Einsatz der eigenen knappen Devisen und der Stärkung der Finanzbasis durch ausländisches Kapital begründet. Auch ergaben sich durch die indische Politik der Importsubstitution verbunden mit einer quali-

6 Vgl. Becker/Thiel, (Fn. 4), S. 58.

7 Vgl. Jayagovinda, Regulation of Foreign Enterprises in India: An Inquiry into FERA, 1973, in: Indian Journal of International Law 1977, S. 325 (327).

8 Behrens, Kontrolle ausländischer Direktinvestitionen, in: RabelsZ 40 (1976), S. 233 (237). 
tativen Ausweitung des indischen Marktes neue Anreize, die bislang importierten Güter in Eigenproduktion herzustellen. ${ }^{9}$

Die privatwirtschaftlichen Kooperationen in Form von Joint Ventures entwickelten sich nach 1950 durch die Wiederbelebung kriegsbedingt unterbrochener Geschäftsbeziehungen oder durch die Umwandlung von ehemaligen Handelsagenturen in Unternehmen mit Produktionsstätten. Die zahlenmäßige Entwicklung der Genehmigung von Kooperationen (Lizenzverträge, Outright Purchases - dies ist die schlichte Abgabe von Fertigungslizenzen und Zeichnungen gegen eine einmalige Zahlung - und Joint Ventures) stieg tendenziell: Im Jahr 1986 wurden 200 deutsch-indische Kooperationen genehmigt. Hierin spiegelt sich die vergleichsweise liberale Genehmigungspraxis der Regierung Rajiv Gandhis in den letzten Jahren wieder. Allerdings führen nicht alle Genehmigungen zu Gemeinschaftsunternehmen. Unter den 700 bestehenden deutsch-indischen Kooperationen gibt es zur Zeit 181 Joint Ventures, ${ }^{10}$ vorzugsweise im Bereich der chemisch-pharmazeutischen und Elektroindustrie sowie im Maschinen- und Anlagenbau. Auffällig ist dagegen der geringe Anteil privater Gemeinschaftsunternehmen etwa im Bergbau oder bei der größeren Eisen- und Stahlerzeugung. ${ }^{11}$ Die Branchenverteilung deutsch-indischer Gemeinschaftsunternehmen entspricht somit weniger der Industriestruktur Indiens oder der Bundesrepublik, sondern zeigt eindeutig, in welchen Bereichen ausländisches KnowHow und Kapital in Indien erwünscht und möglich ist. Eine Betrachtung der Betriebsgrößen nach Beschäftigten und Umsatz der deutsch-indischen Gemeinschaftsunternehmen läßt erkennen, daß sie ähnlich wie die mit rein indischem Kapital arbeitenden Firmen die gesamte Variationsbreite vom lokalen Kleinbetrieb mit 7 Beschäftigten und 150000 DM Umsatz bis zum marktherrschenden Großunternehmen mit mehr als 9000 Beschäftigten und 150 Mio. DM Umsatz umfassen. Die regionale Verbreitung der Gemeinschaftsunternehmen folgt der üblichen Verteilung und Industriestruktur der jeweiligen Zentren und konzentriert sich auf die industriellen Ballungsgebiete Bombay, Dehli, Bangalore und Calcutta.

\section{Indiens Wirtschaftspolitik}

Grundlegend für die Entwicklung der indischen Wirtschaft - im positiven wie im negativen Sinne - war das 'British Raj‘. Es hinterließ Indien ein funktionierendes parlamentarisches Regierungssystem, eine fähige öffentliche Verwaltung, ein die willkürliche Mogulherrschaft ablösendes Rechtssystem und eine Infrastruktur, die Eisenbahn- und Telegraphennetze sowie Hafen- und Bewässerungsanlagen umfaßte. Auf der anderen Seite drosselten die britischen Kolonialherren die indischen Manufakturen - vor allem deren entwickelte Textilindustrie - durch Zwangsabgaben und Schutzzölle, um den indischen Raum für den Absatz englischer Maschinenprodukte zu nutzen.

9 Vgl. Becker/Thiel, (Fn. 4), S. 60.

10 IGCC, (hrsg.) Indo-German Economic Co-operation 1986, Annual Report, Bombay 1987, S. 20.

11 So die Ergebnisse eigener Untersuchungen. 
Gegen Ende des 19. Jahrhundert beginnt langsam eine gewisse Industrialisierung. Diesen sowohl von Engländern wie von Indern - insbesonderen von Parsees - gegründeten Unternehmen verdankt Indien seine industrielle Grundausstattung, die nach der Unabhängigkeit im Jahre 1947 den Ansatzpunkt einer weiteren Wirtschaftsentwicklung bildete. ${ }^{12}$ In seinem vielzitierten 'Industrial Policy Statement ‘ vom 6. April 1948 stellte der damalige Premierminister Jawaharlal Nehru das Regierungskonzept einer 'Mixed Economy' vor, das die jeweiligen Vorteile der plan- und marktwirtschaftlichen Ordnungsmodelle verwirklichen sollte. ${ }^{13}$ Eine wirtschaftliche Erschließung Indiens - so der während seiner Ausbildung in England durch die staatssozialistischen Thesen Bernard Shaws und der Fabian Society nachhaltig beeinflußte Premierminister - sei nur im Rahmen einer staatlichen Wirtschaftslenkung möglich. Um ein 'Socialist Pattern of Society ${ }^{14}$ zu erreichen, müsse der Staat die Privatwirtschaft kontrollieren und lenken, selbst auch im Rüstungsund Verkehrswesen, in der Bewässerung und in der Stahlindustrie tätig werden und darüber hinaus bestimmte wachstumswichtige Wirtschaftszweige fördern.

Durch die ersten Fünfjahrespläne wurde der Ausbau der Landwirtschaft und der Infrastruktur unterstützt. Später verlagerten sich die staatlichen Investitionen in den Bereich der Schwerindustrie, während die Leichtindustrie - weiterverarbeitende und Konsumgüterindustrie - privaten Investitionen vorbehalten bleiben sollte. ${ }^{15}$ Fortgeschrittene Technologien sowie die Grundstoff- und Investitionsgüterindustrie wurden begünstigt, während personalintensive Produktionsverfahren und die Herstellung von Massengütern vernachlässigt wurden. ${ }^{16}$ Neben den öffentlichen Mitteln und dem indischen Kapitalmarkt wurden auch ausländische Quellen zur Finanzierung dieser Programme miteinbezogen, wobei die multi- und bilaterale Kapitalhilfe in den staatlichen Sektor, die ausländischen Direktinvestitionen fast ausschließlich in den privaten Sektor flossen.

\section{Ausländische Direktinvestitionen}

Indien befürchtet, daß durch die Direktinvestitionen ausländischer Unternehmen die Gefahr einer 'Uberf remdung ${ }^{17}$ entstünde. Diese drücke sich durch einen Konflikt zwischen den politischen Zielen der inländischen Wirtschafts- und Gesellschaftspolitik einerseits und den wirtschaftlichen Interessen der ausländischen Unternehmenstöchter andererseits aus. Indien nimmt deshalb gegenüber ausländischen Direktinvestitionen eine zwiespältige Haltung ${ }^{18}$ ein:

12 Vgl. vor allem Rothermund, Indiens wirtschaftliche Entwicklung, Paderborn 1985, S. 72.

13 Vgl. dazu Petersmann, Wirtschaftsintegrationsrecht und Investitionsgesetzgebung der Entwicklungsländer, Baden-Baden 1974, S. 124.

14 Dazu Kust, Foreign Enterprise in India, 2. Aufl., Bombay 1966, S. 44.

15 Vgl. Rothermund, (Fn. 12), S. 155.

$16 \mathrm{Vgl}$. Zehender, Industrielle Kooperation zwischen Industrie- und Entwicklungsländern: Interessenfelder und Steuerungsmechanismen - Fallstudie BRDtl.-Indien, Berlin 1977, S. 26.

17 Dazu Behrens, (Fn. 8), S. 236 ff.

18 Vgl. auch Zweigert/v. Hofmann, (Fn. 5), S. 204 mit weiteren Hinweisen. 
"Since gaining independence in 1947, India has displayed an ambivalent, often contradictory attitude towards private foreign investment. India has attempted to resolve this fundamental conflict of attitudes by being hospitable to a great deal of foreign investment while at the same time being tough on investment which it considers inimical to its economic growth or inconsistent with its political ideology ". ${ }^{19}$

Einerseits vermittelten Direktinvestitionen dem inländischen Markt ausländisches Kapital und Know-how und beschleunigten durch diese Erweiterung der Produktionsstruktur und des Wettbewerbs die wirtschaftliche Integration. Andererseits wird ihnen vorgeworfen, daß sie zu hohe Gewinne und Lizenzgebühren überwiesen, durch Einsatz kapitalintensiver Technologien nur wenige Arbeitsplätze schafften, kaum inländische Vorlieferanten nutzten, nur zu einem geringen Anteil exportierten und durch ihren monopolitischen Einfluß auf wichtige Wirtschaftssektoren inländische Industriebetriebe verdrängten. Zudem bewirke der Umstand, daß inländische Unternehmen vom Ausland her kontrolliert werden könnten, Verhaltensspielräume, welche diesen es ermögliche, nationale wirtschaftspolitische Maßnahmen zu unterlaufen. So werde eine außenwirtschaftliche Abhängigkeit geschaffen und der Handlungsspielraum der inländischen politischen Entscheidungsträger eingeschränkt. Das Charakteristische dieser Konstellation ist also nicht nur die Unterschiedlichkeit der Ziele, sondern auch die unterschiedlichen Durchsetzungschancen der jeweiligen Interessen.

Vor diesem Hintergrund sind folgende ordnungspolitische Grundsätze für die Behandlung ausländischer Direktinvestitionen zu sehen, welche ebenfalls in dem oben zitierten Industrial Policy Statementr von 1948 enthalten sind:20

(1) Ausländische Direktinvestitionen sind für die Versorgung von Devisen und die Ergänzung von modernen Technologien willkommen.

(2) Eine Gleichbehandlung gegenüber inländischen Investitionen wird grundsätzlich garantiert.

(3) Die Uberweisung von Dividenden und Gewinne sowie die Rückführung von Kapital wird - soweit es die Zahlungsbilanz zuläßt - gestattet.

(4) Eine angemessene Entschädigung wird geleistet, wenn und soweit ausländische Unternehmen im nationalen Interesse enteignet werden.

(5) Das Eigentum und die Kontrolle der Gemeinschaftsunternehmen sollte nach einer begrenzten Zeit in einheimische Hände übergehen.

Diese Grundsätze führten zu einem - in seiner Intensität oft wechselnden - rechtlichen Instrumentarium, das dem ausländischen Investor beträchtliche Beschränkungen in Art und Umfang seiner Tätigkeit auf dem indischen Markt auferlegt. Einen völkerrechtlich verpflichtenden Rahmen für diese innerstaatliche Investitionsgesetzgebung bilden die bilateralen Verträge zwischen der Bundesrepublik Deutschland und Indien.

19 Singhal, Taxing for Development: Incentives Affecting Foreign Investment in India, in: Harvard International Law Journal 14 (1973), S. 50 (51).

20 Vgl. Jayagovinda, (Fn. 7), S. 326; Miller, (Fn. 3), S. 65. 


\section{B. Völkerrechtliche Verpflichtungen}

\section{Bilaterales Kapitalschutzabkommen}

\section{Schutzzweck}

Die Investitionsbereitschaft wird von der Uberzeugung geleitet, daß die Rechtssicherheit und die Kontinuität der Investitionspolitik Indiens gewährleistet bleibt. Maßnahmen wie Transfer- und Devisenkontrollen, langwierige Genehmigungs- und Registrierungsverfahren oder Enteignungen, welche die völkerrechtlichen Prinzipien des Eigentumsrechts mißachten, können die unternehmerische Betätigungsfreiheit erheblich beeinträchtigen. Private Direktinvestitionen zu fördern und zu schützen, ist deshalb das vorrangige Ziel bilateraler Kapitalschutz- oder Investitionsförderungsverträge. Bei $34 \%$ aller in einer Umfrage des BDI untersuchten Investitionen spielten Kapitalschutzverträge eine wichtige Rolle für die Investitionsentscheidung. ${ }^{21}$ Diese bilateralen Investitionsförderungsverträge sind - soweit beim Partner durchsetzbar - nach einheitlichen Grundsätzen ('Mustervertrag`) ausgerichtet. Ihr materieller Rechtsschutz ermöglicht gleichzeitig der Bundesrepublik Deutschland zinsgünstige Kredite über die Kreditanstalt für Wiederaufbau zu gewähren. Investitionen durch die Deutsche Entwicklungsgesellschaft zu tätigen oder Hermes- bzw. Treuarbeit - Garantien für das politische Risiko zu übernehmen. So erleichtern sie es nicht nur dem Bund, Förderungsmaßnahmen zu gewähren, sondern auch diesen Anlagegesellschaften, langfristige Investitionsentscheidungen zu treffen. Diese Verknüpfung von Kapitalschutzvertrag und deutschem Garantiesystem gilt als ein besonderes Charakteristikum der deutschen Vereinbarungen. ${ }^{22}$ Die Bundesvepublik Deutschland und Indien vereinbarten ein solches Ubereinkommen am 15. 10. 1964. ${ }^{23}$

\section{Wesentliche Bestimmungen}

Zunächst werden die Vertragsbegünstigten definiert. Diese sind auf deutscher Seite Kapital- und Personengesellschaften, welche in der Bundesrepublik ihren Sitz haben. Es wird also für die Festlegung der Staatsangehörigkeit einer Gesellschaft an deren Hauptverwaltungssitz angeknüpft. Durch dieses formale Kriterium wird der Kreis der Vertragsbegünstigten auch auf solche Gesellschaften erweitert, die sich in den Händen ausländischer Kapitalanleger befinden, ihren Sitz aber in der Bundesrepublik haben. Dem

21 Vgl. Menck, Steuern und Auslandsinvestitionen - Der Einfluß der Steuerpolitik der Entwicklungsländer auf die Förderungsmaßnahmen der Industrieländer für private Auslandsinvestitionen - dargestellt am Beispiel Indien - BRDtl., Hamburg 1972, S. 64.

22 Vgl. Hahn/Gramlich, Regelungstypen internationaler Investitionen, in: Archiv des Völkerrechts 1983, S. 145, (176).

23 Bundesanzeiger Nr. 235 vom 23. 12. 1964, Ziff. 6 b. 
deutschen Vertragssystem wird deshalb ein 'Multilateralisierungseffekt ${ }^{24}$ zugesprochen. Weiterhin erläutert das Abkommen den Begriff der Kapitalanlagen. Dies sind alle deutschen Vermögenswerte, deren Anlage in Indien in Ubereinstimmung mit den einschlägigen indischen Rechtsvorschriften zugelassen wurden. Dies entkräftet den Vorwurf gegenüber Kapitalschutzverträgen mit Entwicklungsländern, daß trotz der formalen Gleichberechtigung der Vertragspartner eine "unechte Gegenseitigkeit" zwischen ihnen herrsche. Die Unternehmen der Industrieländer nähmen die Schutzrechte des Vertrages in Anspruch, während die Entwicklungsländer das entsprechende Wohlverhalten zu erbringen hätten. Demgegenüber unterstellt das deutsche Abkommen - anders als etwa die amerikanischen Verträge ${ }^{25}$ - die Zulassung deutscher Investitionen ganz der indischen Investitionsgesetzgebung. Dies berücksichtigt das Interesse Indiens, seine Regelungshoheit und Entscheidungsfreiheit darüber zu behalten, ob und welche ausländische Investitionen es zulassen will. Hat es jedoch diese Investitionen einmal genehmigt, so ist es gehalten, sie im Einklang mit den nun folgenden Garantien zu behandeln.

Grundgedanke des Abkommens ist die Gewährung der Wettbewerbsgleichheit. Danach sollen deutsche Investoren nicht ungünstiger behandelt werden als einheimische Unternehmen. Dieser Grundsatz der Inländergleichbehandlung beinhaltet allerdings auch, daß später erlassene indische Gesetze ausländische Kapitalanlagen nachteilig beeinflussen können, sofern Inländer in gleicher Weise davon betroffen sind. ${ }^{26}$ Weiterhin gewährleistet das Abkommen die Rückführung des Kapitals sowie den Transfer der Erträgnisse und des Liquidationserlöses. Allerdings steht der völkerrechtliche Transferschutz unter dem Vorbehalt der innerstaatlichen Modalitäten, die vor erfolgter Kapitalanlage in der Genehmigung festgelegt wurden. Für den möglichen Fall der Enteignung und Verstaatlichung garantiert Indien diese zu unterlassen, solange seine nationalen Entwicklungspläne dies nicht verlangen. Eine Enteignung ist mit den völkerrechtlichen Grundsätzen nur aus 'praktischen Gründen und im nationalen Interesser gegen eine rangemessene und billiger sowie 'unverzüglich` zu entrichtende Entschädigung zulässig. Offen bleibt, ob dieses Abkommen auch Schutz vor 'schleichenden Enteignungen' gewährt, also vor Maßnahmen, die durch ihre langfristige Einwirkung auf die Kapitalanlagen in ihren Auswirkungen einer Enteignung gleichzusetzen sind. ${ }^{27}$ Durch die 'Subrogationsklausel, nach der Ansprüche eines deutschen Unternehmens auf die Bundesregierung übergehen können, wird der Kapitalschutzvertrag mit dem Investitionsrisikosystem verknüpft. Durch die Anerkennung eines innerstaatlichen Rechtsübergangs ermöglicht diese Klausel der Bundesrepublik, bei Eintritt eines Versicherungsfalles Schadensersatzansprüche des Investors gegen das Kapitalimportland geltend zu machen. Ein Garantiefall mit Zahlungsfolge für den Bund tritt allerdings nur dann ein, wenn eine Vertragsverletzung vorliegt, bei der das Schiedsgericht angerufen werden kann. Schließlich erstreckt eine Rückwir-

24 Vgl. Frick, Bilateraler Investitionsschutz in Entwicklungsländern, Berlin 1975, S. 172.

25 Dazu Frick, (Fn. 24), S. 247.

26 Vgl. Goltz, Förderung privater Kapitalanlagen (Direktinvestitionen) in Entwicklungsländern, Köln 1975, S. 62 .

27 So Menck, (Fn. 21), S. 65. 
kungsklausel die Bestimmungen des Abkommens auch auf seit dem 1. 1. 1960 vorgenommene Investitionen - sogenannte 'Altanlagen

\section{Wirkung und Bewertung}

Das Kapitalschutzabkommen zwischen der Bundesrepublik Deutschland und Indien bildet eine Ausnahme unter den von der Bundesrepublik ratifizierten Investitionsförderungsverträgen. Es stellt keinen Staatsvertrag, sondern ein Regierungsabkommen dar. ${ }^{28}$ Von ersterem unterscheidet es sich formal gesehen dadurch, daß in seinem Rubrum als vertragsschließende Partei nicht die Bundesrepublik selbst, sondern die Regierung der Bundesrepublik ${ }^{29}$ steht. Diese Besonderheit erklärt sich aus der Vorgeschichte der Vertragsverhandlungen. Indien schließt grundsätzlich keine Kapitalschutzverträge ab. Dieses Regierungsabkommen entstand ausdrücklich auf Wunsch der Bundesrepublik und der deutschen Industrie. Daraus läßt sich jedoch keine unterschiedliche völkerrechtliche Bindungswirkung ableiten, ${ }^{30}$ vielmehr werden die von den Regierungen vertretenen Staaten als Völkerrechtssubjekte berechtigt und verpflichtet. ${ }^{31}$ Durch ihre Vielzahl und Einheitlichkeit strukturieren und kodifizieren diese Kapitalschutzverträge die gewohnheitsrechtlich geltenden Grundsätze des wirtschaftlichen Fremdenrechts zu einem anerkannten Standard. ${ }^{32}$ Andererseits sollte man die Wirkung dieses Vertragssystems nicht überschätzen: Vom Bund anerkannte Schäden sind in 3 Ländern mit und in 3 Ländern ohne Investitionsförderungsverträge eingetreten. ${ }^{33}$

\section{Doppelbesteuerungsabkommen}

\section{Schutzzweck und Entwicklung}

Einkünfte aus deutsch-indischen Joint Ventures unterliegen grundsätzlich einer Besteuerung nach dem Quellenprinzip in Indien wie auch dem Wohnsitzprinzip in der Bundesrepublik Deutschland. Diese doppelte Besteuerung sucht man durch Doppelbesteuerungsabkommen (DBA) zu vermeiden, welche die Vertragsstaaten in der Anwendung ihres in-

28 Frick, (Fn. 24), S. 172; normalerweise handelt es sich um ratifizierte Staatsverträge, vgl. Jüttner, Rechtsschutz von Investitionen in Entwicklungsländern und Investitionsentscheidung, in: Verfassung und Recht in Ubersee 1976, S. 201 (209).

29 Schmidt-Bleibtreu, Klein, Kommentar zum GG, Berlin 1983, Art. 59, Rn. 24, 25.

30 So aber Berger, Schutz deutscher Kapitalanlagen in Indien, AWB 1965, Nr. 8, S. 2.

31 Schmidt-Bleibtreu, Klein, (Fn. 29), Art. 59 Rn. 25; ebenso allerdings ohne Begründung: Frick, (Fn. 24), S. 172; Goltz, (Fn. 26), a.a.O

32 Jüttner, (Fn. 28), S. 206; Frick, (Fn. 24), S. 243.

33 Jahresbericht 1982 der Treuarbeit, zit. aus: Salow, Bundesgarantien für Kapitalanlagen im Ausland und internationaler Investitionsschutz, München 1984, S. 66. 
nerstaatlichen Steuerrechts beschränken. Die Begrenzung kann darin bestehen, daß jeweils der eine der beiden Vertragsstaaten auf die Besteuerung verzichtet - so die Freistellungsmethode; oder darin, daß die Steuer des anderen Staates auf seine eigene angerechnet wird - so die Anrechnungsmethode. Die Normen des DBA lassen sich als IVerteilungsnormen ${ }^{34}$ des internationalen Verwaltungsrechts bezeichnen, da sie die Steuertatbestände, also gleichsam die Steuerquellen ${ }^{35}$ untereinander aufteilen. Die Bundesrepublik Deutschland und Indien haben ein solches Doppelbesteuerungsabkommen am 18. März 1959 abgeschlossen. ${ }^{36}$ In diesem Abkommen ist - im Unterschied zu sonstigen DBA der Bundesrepublik ${ }^{37}$ - das Besteuerungsrecht des Quellenstaates Indien kaum eingeschränkt worden. Deshalb ist dieses Abkommen schon bei seiner Ratifizierung im Bundestag auf erhebliche Kritik gestoßen. ${ }^{38}$ Erst im Jahre 1973 kam es nach langjährigen deutschen Bemühungen zur Aufnahme erneuter Verhandlungen. Am 18. März 1985 (!) konnte ein Änderungsprotokoll, welches die Begrenzung der Quellenbesteuerung in Indien noch in wesentlichen Punkten zu verbessern sucht, ratifiziert werden. Dabei waren - so die Denkschrift zum Änderungsprotokotoll ${ }^{39}$ - die finanz- und entwicklungspolitischen Interessen der Republik Indien als einem Entwicklungsland einerseits und die wirtschaftspolitischen Interessen der Bundesrepublik Deutschland als einem Industriestaat andererseits zu berücksichtigen. Während die Bundesrepublik als kapitalgebendes Land die Steuerpflicht in Indien für die dort getätigten Investitionen klar und eindeutig umgrenzen möchte, will Indien als kapitaleinführendes Land demgegenüber ein Besteuerungsrecht für die nach der Bundesrepublik fließenden Einkünfte möglichst uneingeschränkt aufrechterhalten.

\section{Wesentliche Bestimmungen}

Deutsch-indische Joint Ventures sind rechtlich gesehen als 'Private Limited Companies oder 'Public Limited Companies` indische Gesellschaften. Deshalb unterliegen sie dem indischen Steuerrecht, das wiederum durch das deutsch-indische Doppelbesteuerungsabkommen verdrängt werden kann. Folgende Steuerarten und Bestimmungen des Doppelbesteuerungsabkommens sind von Bedeutung:40

34 Vgl. Vogel, Doppelbesteuerungsabkommen, Kommentar, München 1983, DBA, Einl., Rdnr. 16, 24.

35 BFH, BStBl 1965, 352 (353).

36 BGBI 1960 II, 1829.

$37 \mathrm{Vgl}$. Debatin, Das deutsch-indische Doppelbesteuerungsabkommen als steuerliche Grundlage für Investitionen in Indien, in: AWD 1961, S. 6.

38 Vgl. Entschließung des BT vom 6. 4. 1960.

39 In: BT-Drucksache 10/2668, S. 19.

40 Trotz Verwendung aktuellster Literatur und zahlreicher Erkundigungen durch den Verfasser können einige der Zahlenangaben sich wieder geändert haben. 


\section{a. Besteuerung der Unternehmensgewinne}

Die Unternehmensgewinne deutsch-indischer Joint-Ventures unterliegen zunächst der indischen Einkommenssteuer. Diese beträgt 52,5\% bis 68,25\%.41 Die Besteuerung dieser Gewinne, der wichtigsten Einkunftsart des DBA-Rechts, ${ }^{42}$ erfolgt nach dem Betriebsstättenprinzip. Danach fallen Einkünfte nur dann unter die indische Steuerhoheit, wenn sie einem 'Permanent Establishment، in Indien zurechenbar sind. Eine solche Betriebsstätte liegt schon bei einer Montage oder Bauausführung, nach dem Revisionsprotokoll von 1985 allerdings erst ab einer Dauer von sechs Monaten, ${ }^{43}$ vor. Weiterhin erläutert das Abkommen, wie diese Gewinne den einzelnen 'Permanent Establishments` zuzuordnen und welche Ausgaben abzugsfähig sind. ${ }^{44}$

\section{b. Besteuerung der Dividenden}

Wenn deutsch-indische Joint Ventures in Indien Dividende erklärten und ausschütteten, so erlaubte das DBA von 1959 eine Besteuerung durch beide Vertragsstaaten. Damit sah das Abkommen entgegen der sonstigen Vertragspraxis der Bundesrepublik keine Begrenzung oder Aufhebung der Quellenbesteuerung in Indien vor. Diese Regelung, die eine Doppelbesteuerung nicht zu vermeiden sondern eher zu bestätigen schien, fiel somit 'vollkommen aus dem Rahmen der übrigen deutschen Abkommen'. ${ }^{45}$ Ublicherweise ist eine Aufteilung der Besteuerung von Dividenden zwischen Quellen- und Wohnsitzstaat vorgesehen. Die Begrenzung des Quellensteuersatzes wird mit einer Anrechnungs- oder Befreiungsverpflichtung des Wohnsitzstaates kombiniert. Grundsätzlich gilt in Indien für ausgeschüttete Dividenden ein Steuersatz von $25 \% .{ }^{46}$ Das Revisionsprotokoll von 1985 senkt nun die Quellensteuer für Dividenden von Neuanlagen auf $15 \%$. Diese Begrenzung der indischen Besteuerungsbefugnis ergibt sich allerdings nur für Dividenden, deren zugrundeliegendes Gesellschaftskapital erst nach dem Inkrafttreten des Revisionsprotokolls, also nach dem 10. 8. 1985, ausgegeben wurde. Der Sinn dieser Regelung wurde offenbar aus indischer Sicht darin gesehen, einmal nach Indien vergebenes Kapital nicht noch nachträglich zu begünstigen ${ }^{47}$ Eine weitere in diesem Zusammenhang wichtige Regelung betrifft das Schachtelprivileg. Nach dem Abkommen von 1959 waren Dividenden, welche deutsche Kapitalgesellschaften aus einer Mindestbeteiligung von $25 \%$ an einem Joint Venture in Indien bezogen, grundsätzlich von der deutschen Steuer frei. ${ }^{48}$

41 Advani, Direct Taxes in India, in: Indo-German Economy 1986, S. 54.

42 So Vogel-Hemmelrath, (Fn. 34), Art. 7, Rdnr. 3.

43 Jonas, Deutsch-indisches Revisionsprotokoll, in: Deutsch-indisches Doppelbesteuerungsabkommen, hrsg. IGCC, Bombay 1986, S. 55.

44 Dazu näher Advani, Taxation and Turn-Key Projects, in: Indo-German Economy 1986, S. 49.

45 So wörtlich Vogel-Tischbirek, (Fn. 34), Art. 10, Rdnr. 84.

46 Advani, (Fn. 44), S. 32.

47 So Jonas, (Fn. 43), S. 63.

48 Vgl. Debatin, (Fn. 37), S. 11. 
Das Revisionsprotokoll von 1985 ermöglicht das inzwischen in der Bundesrepublik geänderte Schachtelprivileg schon bei einer Mindestbeteiligung von $10 \% .^{49}$

\section{c. Besteuerung von Lizenzgebühren}

Lizenzgebühren sind Vergütungen, die für die Benutzung von Erfahrungen, Urheberund gewerblichen Schutzrechten geleistet werden. Diese werden entweder durch eine einmalige Zahlung ('Lump Sum`) und/oder durch regelmäßig wiederkehrende Leistungen ('Royalties') entgolten. Entgegen dem in sonstigen DBA enthaltenem Grundsatz der Wohnsitzbesteuerung für Lizenzgebühren gab das Abkommen von 1959 deren Quellenbesteuerung in Indien völlig frei, welche allerdings auf die Wohnsitzsteuer angerechnet werden konnte. ${ }^{50}$ Diese betrug für Royalties $40 \%$, für Lump Sums $20 \%$. Für das Finanzjahr 1987/88 ist nun eine einheitliche Besteuerung der Royalties wie der Lump Sums in Höhe von $30 \%$ vorgesehen. ${ }^{51}$ Nach dem Revisionsprotokoll von 1985 gilt darüber hinaus eine Sonderregelung, wonach die Quellensteuer auf Lizenzgebühren nicht $20 \%$ übersteigen darf. Im Ergebnis ergibt sich daraus ein Besteuerungsrecht des Quellenstaates Indien für Leistungen, welche im wesentlichen in der Bundesrepublik erbracht wurden..$^{52}$

\section{d. Besteuerung von Zinsen}

Nach dem indischen Steuerrecht sind Zinsen für Kredite von ausländischen Unternehmen mit $25 \%$ zu besteuern. Nach dem Abkommen von 1959 beträgt die Quellensteuer $10 \%$, wenn der Kredit von einer deutschen Bank, $15 \%$ wenn er von einem sonstigen deutschen Investor gewährt wurde. ${ }^{53}$ Zinsen für Kredite der Deutschen Bundesbank, der Kreditanstalt für Wiederaufbau oder der Deutschen Entwicklungsgesellschaft sind quellensteuerfrei.

\section{e. Steuervergünstigungen}

Um einen Anreiz für Investitionen in Indien zu bieten, sieht das Income Tax Law bestimmte Steuererleichterungen vor. Bisher wurden Doppelbesteuerungen dadurch vermieden, daß die in Indien entrichtete - ermäßigte - Steuer auf den Betrag der deutschen Steuer angerechnet wurde. Das Revisionsprotokoll von 1985 regelt die Anrechnung indischer Steuererleichterung neu: Es wird die übliche Steuer ohne Rücksicht auf gewährte Erleichterungen angerechnet. Durch diese Anrechnung der fiktiv erhobenen, statt der

Malegam, Indisches Steuerrecht, in: Deutsch-indisches Doppelbesteuerungsabkommen, hrsg. IGCC, Bomby 1986, S. 84.

50 Vgl. Vogel-Pöllath, (Fn. 34), Art. 12, Rdnr. 30, 70.

51 Advani, (Fn. 41), S. 54.

52 So Vogel-Pöllath, (Fn. 34), Art. 12, Rdnr. 13.

53 Advani, Taxation and Different Forms of Collaboration, in: Indo-German Economy 1986, S. 32 (32). 
faktisch bezahlten Steuer werden die Vergünstigungen der indischen Steuer im wesentlichen Umfang an den deutschen Steuerpflichtigen weitergegeben: Erstens durch die Steuererleichterung im Anlagestaat und zweitens durch die fiktive Anrechnung im Wohnsitzstaat des Empfängers. ${ }^{54}$ Der Aufkommensverlust dieser 'doppelten Steuersenkung wird allerdings nur in DBA mit Entwicklungsländern eingenommen. ${ }^{55}$

\section{Indiens Investitionsgesetzgebung}

Für Gemeinschaftsunternehmen mit ausländischer Kapitalbeteiligung ist das 1973 erlassene 'Foreign Exchange Regulation Act' (im folgenden FERA 1973 genannt) besonders wichtig geworden. Das Ziel dieser Bestimmung ist die Erhaltung der knappen Devisenreserven des Landes sowie deren angemessene Nutzung im 'nationalen Interesser. Dies soll durch eine Kontrolle der ausländischen Investitionen erreicht werden. Dafür ergeben sich grundsätzlich drei Ansatzpunkte: ${ }^{56}$

(I) Kontrolle der Eigentumsverhältnisse

(II) Kontrolle der Unternehmensaktivitäten

(III) Kontrolle der Kapital- und Güterbewegungen

Dieses dem öffentlichen Wirtschaftsrecht zuzuordnende Instrumentarium faßt Funktionen des Gesellschafts-, Niederlassungs- und Außenwirtschaftsrechts zusammen. Letzteres wird noch durch umfangreiche Genehmigungsbestimmungen ergänzt.

\section{Kontrolle der Eigentumsverhältnisse}

Diese Regelungen sollen verhindern, daß ausländische Unternehmen einen beherrschenden Einfluß in den Organen des inländischen Gemeinschaftsunternehmens ausüben. Ansatzpunkte können dabei sowohl die Gesellschaftsversammlung als auch die Leitungsund Kontrollorgane sein. FERA 1973 setzt vor allem bei der Gesellschaftsversammlung an. Artikel 29 bestimmt, daß

"a company which is not incorporated under any law enforced in India or in which the non-resident interest is more than forty per cent . . . shall not, except with general or special permission of the Reserve Bank of India, carry on or establish in India a branch office or other place of business for carrying on any activity of trading, commercial or industrial nature". ${ }^{57}$

54 Vgl. Menck, (Fn. 21), S. 99.

55 So der Finanzausschuß, in: BT-Drucksache, III, 1763.

56 Dazu im folgenden: Jayagovinda, (Fn. 7), S. 225 f.; Behrens, (Fn. 8), S. 224 f.; Petersmann, (Fn. 13), S. 126 f.

57 Zit. aus Kapoor, Taming the Multinationals in India, in: Journal of World Trade Law 1979, S. 171 (171). 
Die Beteiligung von Ausländern am Aktienkapital wird grundsätzlich auf maximal $40 \%$ festgelegt, FERA 1973 will der Gefahr einer 'Uberfremdung den Prozeß einer Indianization $^{58}$ entgegensetzen. Diese soll eine Geschäftspolitik gewährleisten, welche `nationale Zielerstärker beachtet. Die Beschränkung gilt nicht nur für neu zu gründende Unternehmen, sondern auch für bereits bestehende Gesellschaften mit ausländischer Kapitalbeteiligung. Letztere sind gehalten, sich den neuen Richtlinien nachträglich anzupassen. Das Kriterium des ausländischen Besitzanteils bis zu $40 \%$ geht von der Vermutung aus, daß ein solcher Eigentümer auf die Geschäftspolitik eines Unternehmens noch nicht vollständig Einfluß nehmen könne. ${ }^{59}$ Die indische Mindestbeteiligung hat $26 \%$ zu betragen. Unternehmen, deren ausländischer Besitzanteil weniger als $40 \%$ beträgt, fallen nicht unter das FERA 1973. Ihnen wird garantiert, grundsätzlich wie indische Unternehmen behandelt zu werden.

Die Kontrolle der Eigentumsverhältnisse wird durch eine weitere Bestimmung aus dem 'Companies Act ergänzt. Sie regelt die Veräußerung und den Erwerb von Aktien. Danach sind indische Aktien nummerierte Anteilsrechte, die begrifflich die Ausstellung einer Urkunde nicht voraussetzen. Damit der Eigentümer sein Anteilsrecht beweisen kann, wird ihm nach der Zuteilung der Aktien ein Anteilsschein ausgestellt. ${ }^{60}$ Die Ubertragung von Aktien setzt nun voraus, daß die neuen Gesellschafter in das Register der Gesellschaft eingetragen werden. Die Satzung bindet den Antrag auf Eintragung und damit die Veräußerung dieser Aktien an die Zustimmung der Gesellschaft. ${ }^{61}$ Dies entspricht einer Vinkulierung von Namensaktien. Die Satzung kann den 'Board of Directors` verpflichten, die Zustimmung zum Erwerb von Aktien durch Ausländer zu versagen.

Ein weiterer Ansatzpunkt sind die Leitungs- und Kontrollorgane des Joint Ventures. Zur 'Indianization` gehört auch die Ausbildung, Einstellung und Beteiligung indischer Führungskräfte in Gemeinschaftsunternehmen. Dadurch soll der Einfluß auf Entscheidungsprozesse gesichert werden. Eine entsprechende Kontrolle ist leicht durchzuführen, da die Arbeits- und Aufenthaltserlaubnis ausländischer Mitarbeiter regelmäßig neu beantragt und genehmigt werden muß.62

Von der Konsumgüterproduktion sind ausländische Kapitalgeber im wesentlichen ausgeschlossen. Die von ihnen produzierten 'westlichen Konsumgüter seien mit den Zielen einer sozialistischen Gesellschaft unvereinbar, da sie nur für den Geschmack und die Kaufkraft einiger weniger gedacht seien, soziale Unterschiede förderten und traditionelle Werte untergrüben. ${ }^{63}$ Von Bereichen wie Banken, Handel und Versicherungen sind sie ebenfalls ausgeschlossen. Ausländische Investitionen sollen weniger als finanzielle, son-

58 Vgl. dazu Miller, (Fn. 3), S. 87.

59 So Jayagovinda, (Fn. 7), S. 329.

60 Warekar, Gesellschaftsrecht in Indien, hrsg. IGCC, Bombay 1984, S. 52.

61 Sevin, Das Recht der indischen Kapitalgesellschaften, 2. Aufl., hrsg. Bundesstelle für Außenhandelsinformation, Köln 1967, S. 24.

$62 \mathrm{Vgl}$. Kumar, Führungsprobleme internationaler Gemeinschaf tsunternehmungen in den Entwicklungsländern, Meisenheim 1975, S. 29.

63 Dazu Miller, (Fn. 3), S. 72. 
dern vielmehr als technologische Unterstützung dienen. Der Handel mit nicht selbst hergestellten Produkten ist FERA-Gesellschaften aus den Erfahrungen mit den früheren auf die Kolonialzeit zurückgehenden 'Managing Agencies` untersagt worden. Diese waren rechtlich und wirtschaftlich selbständige Firmen, welche die Führung einer oder mehrerer Unternehmen auf vertraglicher Basis gegen eine vereinbarte Gewinnbeteiligung oder Jahresgebühr übernahmen. ${ }^{64}$ Uber die 'Agencies` dominierten vor allem britische Firmen bis 1947 ganze Industriezweige, wie etwa Jute, Tee, Eisen oder Stahl, ohne daß sie das entsprechende Kapital hätten einsetzen müssen. Die Untersagung des Handelns hatte für einige Joint Ventures einschneidende Folgen. Bei Anlagen und Systemen mußte die Produktpalette ergänzt werden. Auch hatten deutsche Unternehmen mehrere Produktionsgesellschaften gegründet, deren Produkte von der indischen Muttergesellschaft insgesamt vermarktet wurden. Die Produktionsgesellschaften mußten sich nun eigene Vertriebsorganisationen schaffen.

\section{Kontrolle der Unternehmensaktivitäten}

Artikel 29 selbst stellt keine Kriterien auf, nach denen bestimmten Unternehmen mit einer ausländischen Beteiligung von mehr als $40 \%$ eine Ausnahmegenehmigung erteilt werden kann. Diese sind jedoch in sogenannten 'Guidelines' niedergelegt, welche seit 1974 vom Industrieministerium herausgegeben werden. Die Richtlinien knüpfen an den Vorgang der Beteiligungsübernahme an und ermöglichen eine Beteiligung von $40 \%$, $51 \%$ und $74 \%$. Die Kriterien für diese verschiedenen Prioritätsklassen sind:

(1) Non availability of comparable indigenous resources

(2) Essentiality in the sense of contributing to development

(3) Effects on balance of payments ${ }^{65}$

Die FERA 1973 Richtlinien erlauben ausländische Investitionen bis zu 74 \% nur dort, wo die Produktion der einheimischen Industrien noch ungenügend ist, deren Technologie unterentwickelt ist und diese Investitionen bestehende indische Firmen nicht verdrängen, Gemeinschaftsunternehmen sind dann erwünscht, wenn sie wesentlich zum Technologietransfer oder zur Exportmarkterschließung beitragen. Das Genehmigungsverfahren sieht eine flexible Anwendung dieser Kriterien vor, um besonderen Umständen ron merits` bei einzelnen Anträgen gerecht werden zu können. Im wesentlichen führten zwei Uberlegungen zu diesen Kriterien:

Indien hat in Bereichen wie etwa Schwerchemie, Verhüttungswesen, Kraftwerks- und Fahrzeugbau noch keine genügend entwickelte Industrie. ${ }^{66} \mathrm{Da}$ es einerseits die im eigenen Land nicht verfügbaren hochwertigen Güter, Technologien und Rohstoffe einführen muß, denen aber andererseits nicht genügend Devisen aus Exporterlösen gegenüberste-

64 Vgl. Kumar, (Fn. 62), S. 223.

65 Jayagovinda, (Fn. 7), S. 327.

66 Aggarwal, Law Relating to Foreign Companies and Foreign Collaborations, Bombay 1986, S. 10. 
hen, leidet Indien unter einem ständigen Mangel an Devisen. ${ }^{67}$ Um das Defizit der Zahlungsbilanz nicht größer werden zu lassen, beschränkt und konzentriert man ausländische Investitionen auf diejenigen Bereiche, in denen die einheimische Produktion noch unterentwickelt und 'Sophisticated Technology' somit erwünscht ist. Durch einige Liberalisierungen in den letzten Jahren gibt es nun wieder mehr 'Core Areas', in denen investiert werden darf. ${ }^{68}$

Auch sollen diese Bestimmungen eine protektionistische Wirkung entfalten. Den Ergebnissen zweier Untersuchungskommissionen zufolge ${ }^{69}$ würden gerade ausländische Unternehmen durch ihre überlegene Technik und Produktionseffizienz kleinere indische Firmen vom Markt verdrängen. Die darauf 1969 erlassene Kartell- und Antimonopolgesetze sowie die FERA-Richtlinien wollen indische Small-Scale-Industriesı schützen. Dies gilt vor allem für Bereiche wie landwirtschaftliche Maschinen, Büroausstattungen oder Gebrauchtwagen.

\section{Kontrolle der Kapital- und Güterbewegungen}

Durch die hier angewandten Bestimmungen soll der gesamte Waren- und Dienstleistungsverkehr sowie der Kapital- und Zahlungsverkehr erfaßt werden. Grundsätzlich ist der Devisenverkehr frei. Um jedoch die Zahlungsbilanz vor übermäßigen Dividenden-, Gewinn- oder Lizenzgebührüberweisungen zu schützen, wurden gewisse Kontroll- und Begrenzungsmaßnahmen eingeführt. ${ }^{70}$ Deshalb stehen diese Zahlungsbewegungen unter einem Genehmigungsvorbehalt der 'Reserve Bank of India', an welche die Kompetenz zur Erteilung einer Erlaubnis delegiert wurde. Der Genehmigungsvorbehalt dient schon der Uberprüfung, ob die auf diese Zahlungen anfallenden Steuern bereits gezahlt wurden. Es steht in freiem Ermessen der Reserve Bank of India, ob sie eine solche Genehmigung gewährt oder versagt. Rechtsgrundlagen sind neben FERA und deren Richtlinien das 'Industries (Development and Regulation) Act', 1951; das 'Monopolies and Restrictive Trade Practices Actı, 1969; das 'Imports and Exports (Controll) Act 1947 und das 'Companies Act‘, 1956. Die indische Regierung bevorzugt im allgemeinen Lizenzverträge gegenüber Joint Ventures. Insbesondere ist sie an sogenannten 'Outright Purchases interessiert, also der schlichten Abgabe von Fertigungslizenzen und Zeichnungen gegen eine einmalige Zahlung ohne weitergehende Zusammenarbeit mit dem ausländischen Partner. Die Lizenzgebühren können entweder in einer Pauschalsumme ('Lump Sum`) oder in regelmäßig wiederkehrenden Zahlungen ('Royalties`) geleistet werden. Die FERA-Richtlinien sehen nun vor, daß die 'Lump Sum` in drei Raten gezahlt werden

67 Dazu Krishnaswamy, Foreign Exchange Management in India and the Philippines, in: Indian Journal of Public Affairs 1980, S. 21 (21).

68 Vgl. IGCC, (Fn. 10), S. 109.

69 The Hazari and Dutt inquiriess.

70 Vgl. Agrawal, Business Collaborations in India, 4. Aufl., Bombay 1986, S. 39. 
soll, während die 'Royaltiesı nicht mehr als $5 \%$ des Umsatzes betragen und deren Laufzeit nicht länger als 10 Jahre dauern darf.

Für Investitionen in Joint Ventures gilt, daß sie nicht mit dem Import von Gütern, Lizenzzahlungen oder Warenzeichenvergütungen aufgerechnet bzw. verknüpft werden dürfen, sondern in bar erfolgen müssen. ${ }^{71}$ Dadurch möchte die indische Regierung bezwekken, daß Joint Ventures den benötigten Maschinenpark in Indien beziehen. Will dagegen ein deutsches Unternehmen sich von seiner Beteiligung lösen, so ist eine Genehmigung erforderlich, wenn der Verkauf der Anteile $10 \%$ des Stammkapitals übersteigt. Die Reserve Bank of India prüft die Eignung des indischen Käufers, die Angemessenheit des Ubernahmepreises, die mögliche Änderung in der Zusammensetzung des 'Board of Directors` und schlägt eine Uberweisung in Raten vor.

Oft werden die für die Produktion notwendigen Güter und Maschinen, Rohstoffe und Halbfabrikate aus dem Ausland - meist von einem Mutterunternehmen - eingeführt. Hierfür ist ebenfalls eine Genehmigung nötig. Dadurch gewinnt die Regierung einmal die Kontrollmöglichkeit darüber, daß bei entsprechendem Angebot an indischen Maschinen diesen der Vorzug gegeben wird. Zum anderen erhält sie dadurch die Gelegenheit, eine Kontrolle über die eingesetzten Preise auszuüben. ${ }^{72}$ Auch darf die vertragliche Vereinbarung keine Exportklausel beinhalten und muß weitere Sublizenzverträge erlauben. Diese Maßnahmen führten in der Vergangenheit zusammen mit der damit verbundenen bürokratischen Handhabung ('red tape`) dazu, daß es beim Aufbau und bei der Produktionsausstattung der Joint Ventures häufig zu beträchtlichen Verzögerungen und Produktionseinschränkungen kam.

\section{Wirkung und Bewertung}

Die Wirkungen der FERA-Bestimmungen von 1973 betrafen 814 Unternehmen. ${ }^{73} 43 \%$ verringerten ihren Anteil auf die erforderliche Größe, wobei einige bei einer ausländischen Beteiligung von $51 \%$ und $74 \%$ bleiben durften. $51 \%$ blieben unverändert, da sie vorher schon unter $40 \%$ lagen. $6 \%$ schließlich zogen es vor, sich aus Indien zurückzuziehen. Ausschlaggebend für sie war - wie etwa IBM oder Coca Cola - die Befürchtung, daß die geringe Beteiligungsquote die Geheimhaltung der Herstellungsverfahren gefährde. ${ }^{74}$

Die gegenüber den 50er und 60er Jahren nachlassende Neigung deutscher Unternehmer, in Indien zu investieren, wurde nicht zuletzt durch diese Reglementierung nachhaltig beeinflußt. Sie regelt die Rechtsbeziehungen zwischen dem Gastland und dem Auslandsunternehmen weniger unter dem Gesichtspunkt unveränderter Eigentumspositionen, son-

71 Vgl. Desai, Non-Residents: Investments, Incentives \& Tax Planning, Delhi 1986, S. 31.

72 Oldenbruch, Gründung von Partnerschaftsunternehmen in der indischen Union, Diss., Köln 1963, S. 59.

73 Dazu Miller, (Fn. 3), S. 86.

74 Vgl. Chauduri, FERA: Appearances and Reality in: Econ. \& Pol. Weekly Bombay 1979, S. 734 (736). 
dern ordnet diese vielmehr den Prioritäten nationaler Wirtschaftspolitik unter. Die auffallende Massierung der deutsch-indischen Joint Ventures mit einem Anteil von 30-40\% Auslandskapital zeigt die spürbaren Auswirkungen der FERA-Bestimmungen. Die Verminderung des deutschen Anteils wurde entweder durch Kapitalerhöhung seitens des indischen Partners vollzogen oder aber durch Umwandlung der Joint Ventures von einer 'Private Limited، in eine 'Public Limited Company', verbunden mit der Ausgabe zusätzlicher Aktien.

Auf Unternehmensebene bleibt offen, ob der Eigentümerwechsel eine entsprechende Verstärkung der indischen Kontrolle und Beachtung 'nationaler Belanger bewirkte. Die meisten Unternehmen verringerten ihre ausländische Beteiligung durch freien Verkauf der Aktien an der Börse. Da jeder Einzelne nur eine begrenzte Anzahl von Aktien erwerben durfte, wurden die Aktien breit gestreut. Diese von der Regierung erwünschte Förderung des Volksvermögens hatte freilich einen Nachteil:

'The dispersal of shares among thousands of small investors who generally are uninterested in the management of the company leads to the result that as little as $5 \%$ equity wil ensure control. ${ }^{75}$

Wo sich nicht die erforderliche Anzahl von Käufern fand, gelangten die Aktien gebündelt in den Besitz großer Finanzinstitutionen oder Familienunternehmen. So behält selbst bei einer Minderheitsbeteiligung das deutsche Unternehmen durch seine finanziell-technologische Uberlegenheit oder eine Streuung der restlichen Unternehmensanteile über eine große Zahl lokaler Aktionäre den von ihm gewünschten Einfluß auf die Unternehmensführung.

Auf rechtlicher Ebene fallen die vagen Kriterien wie 'national interest', 'contributing to development' oder 'sophisticated technology' auf. Diese Generalklauseln genügen kaum einem Bestimmtheitsgrundsatz von Ermächtigungsnormen ${ }^{76}$ und stellen die Entscheidung auf Zulassung eines Joint Ventures einem nicht justiziablen Ermessen ${ }^{77}$ der Behörden anheim.

Auch auf gesamtwirtschaftlicher Ebene bleibt fraglich, ob FERA seine angestrebten Ziele erreichen konnte. Der Verkauf der ausländischen Beteiligungen absorbierte in beachtlichem Maße indisches Kapital. Dieses wurde dadurch in bereits bestehende Unternehmen abgelenkt, ohne daß letztendlich eine Förderung neuer Unternehmen entstand. ${ }^{78}$ Zudem verwandten viele dieser Unternehmen, nun als 'Domestic Enterprises' angesehen, den Verkaufserlös dazu, in Bereiche zu expandieren, die ihnen vorher als 'Foreign Enterprises` verschlossen waren. Dort wiederum verdrängten sie z. T. kleinere Firmen.

75 Chauduri, (Fn. 74), S. 740.

76 So Petersmann, (Fn. 13), S. 265.

77 Vgl. Hahn/Gramlich, (Fn. 22), S. 209.

78 Kapoor, (Fn. 57), S. 174. 


\section{Vertragliche Vereinbarungen}

\section{Indisches Gesellschaftsrecht}

Das Recht der indischen Kapitalgesellschaften ist im 'Companies Act' von 1956 geregelt. Es ist auf alle Gesellschaften, die in Indien geschäftlich tätig werden, anwendbar und beruht im wesentlichen auf dem englischen Gesellschaftsrecht. Dies gilt nicht nur für die Gesetzgebung, sondern auch für seine Auslegung, da die Entscheidungen britischer Gerichte subsidiär herangezogen werden können. Tragendes Prinzip war deshalb die Vertragsfreiheit ${ }^{79}$ der Gründer und Mitglieder einer Gesellschaft. Verschiedene Änderungen der letzten Jahre hatten allerdings zur Folge, daß man sich von diesem Prinzip löste, und Aufgaben und Arbeitsweise einer Gesellschaft umfassend gesetzlich regelte. Das 'Companies Act ' von 1956 sowie seine darauffolgenden 'Amendment Acts' haben einen außerordentlichen Umfang. Sie regeln weitgehend alle denkbaren Einzelfälle. Diese Gestaltung wurde deshalb für nötig erachtet, weil die Umgehung von Gesetzen nicht unüblich ist. So hoffte man, durch genaue Einzelregelungen die - oft gerichtliche - Klärung der Rechtslage zu verkürzen und zu vereinfachen.

Für deutsch-indische Gemeinschaftsunternehmen stellen die 'Public Company Limited by shares und die 'Private Company limited by shares` die übliche Gesellschaftsform dar. Erstere ist der deutschen Aktiengesellschaft, letztere der deutschen Gesellschaft mit beschränkter Haftung vergleichbar. Beides sind selbständige, von ihren Mitgliedern unabhängige juristische Personen. Die Beteiligung an einer solchen Gesellschaft oder die Gründung einer solchen mit einem indischen Partner ist genehmigungsbedürftig und nur in Verbindung mit einem Know-How-Transfer möglich. Das Companies Act behandelt beide Formen nicht nebeneinander, sondern entwickelt die 'Private Company aus der 'Public Company' durch erweiterte oder verminderte Erfordernisse, die verstreut in den einzelnen Abschnitten aufgeführt werden.

\section{Gründung eines Gemeinschaftsunternehmens}

Zunächst legen der deutsche und der indische Partner in einer sog. Grundlagenvereinbarung, einer Art Konsortialvertrag zwischen den Beteiligten, ${ }^{80}$ die Verfassung ihres Gemeinschaftsunternehmens fest. Wesentliche Punkte wie Ziele, Willensbildung, Finanzierung oder zukünftige Tätigkeitsfelder und Produktionsaufgaben ihrer Zusammenarbeit werden darin umschrieben. Diese im angelsächsischen Rechtskreis üblichen 'Heads of Agreement ${ }^{81}$ enthalten somit die in den Vorverhandlungen entworfene unternehmeri-

79 Singhania, Indisches Gesellschaftsrecht für ausländische Investoren, in: Dt.-Ind. Wirtschaft 6/86, S. 3.

80 Vgl. Ebenroth, MüKomm, München $1984 \mathrm{ff}$., nach Art 10 EGBGB, Rn. 371.

81 Dazu Schaub, Gemeinschaftsunternehmen in: Handbuch der Unternehmenszusammenschlüsse, hrsg. Sölter, Zimmer, 1972, S. 179 (182). 
sche Grundrichtung. Dieser Gründungsvorvertrag wird dann durch die eigentliche Gründung der juristischen Person gleichsam vollzogen. Dafür sind nach den indischen Rechtsvorschriften sowohl ein 'Memorandum of Association' wie auch die 'Articles of Association' erforderlich. Das 'Memorandum of Association' präzisiert die unternehmerischen Grundlinien unter Beachtung der gesellschaftsrechtlichen Erfordernisse in juristischer Form. Dieser oft auch als 'Capital Collaboration Agreement ‘ bezeichnete Gesellschaftsvertrag regelt im wesentlichen Verfassung, Zweck, Gegenstand und Stammkapital des Gemeinschaftsunternehmens. Er wird durch die 'Articles of Association` ergänzt, welche eine Art Verfahrensordnung darstellen. Teils bestätigen sie gesetzlich vorgegebene Erfordernisse; so schränkt diese Satzung gemäß Section 27 des 'Companies Acts` die Ubertragbarkeit von Aktien ein, begrenzt bei einer 'Private Company` die Zahl der Gesellschafter auf 50 und untersagt öffentliche Zeichnungsaufforderungen für Aktien. Teils enthält diese Satzung auch Regelungen, deren Gestaltung ihr durch das 'Companies Act' aufgegeben sind; so befaßt sie sich mit der Geschäftsverteilung, den Beziehungen der Mitglieder untereinander und zur Gesellschaft und einem möglichen Minderheitenschutz.

Die Gründung ist nach indischem Aktienrecht mit der Eintragung der Gesellschaft im Handelsregister abgeschlossen. ${ }^{82}$ Diese Eintragung führt allerdings nur zur Bildung einer juristischen Person, nicht aber zur Aufnahme der wirtschaftlichen Geschäftstätigkeit. ${ }^{83}$ Bis zu diesem Zeitpunkt abgeschlossene Verträge sind schwebend unwirksam. Erst nach erfolgter Einbringung des Kapitals erfolgt die Zulassung zur Betriebsaufnahme.

Bemerkenswert ist, daß eine 'Private Company', an deren eingezahltem Kapital eine andere Gesellschaft - 'Private Company' oder 'Public Company' - sich mit mehr als $25 \%$ beteiligt, kraft Gesetz sich in eine Public Company verwandelt. Während für eine `Private Company' weniger Beschränkungen bestehen, unterliegt die nun in eine 'Public Company، umgewandelte Gesellschaft u. a. strengeren Vorschriften für den Jahresabschluß. Dieser Regelung liegt das öffentliche Interesse zugrunde, die nun sich auf dem Kapitalmarkt finanzierende Gesellschaft zu überwachen. ${ }^{84}$

Im folgenden wird auf die wesentlichen vertraglichen Bestimmungen eines 'Capital Collaboration Agreements' und ihrer Ausformung in der Satzung der 'Articles of Association r eingegangen.

\section{Beteiligungsverhältnisse}

Besonders wichtig ist die Regelung der finanziellen Beteiligungsverhältnisse. Die von den Partnern in scash or kind eingebrachten Einlagen werden daher genau beschrieben. Die Frage, wie diese zu bewerten sind, ist darüber hinaus für Anteile, die durch eine Kapital-

82 Vgl. Oldenbruch, (Fn. 72), S. 76.

83 Vgl. Warekar, (Fn. 60), S. 20.

84 So Sevin, (Fn. 61), S. 4. 
erhöhung neu ausgegeben wurden, von Bedeutung. Hier wird meist eine Klausel wie 'Fair Market Value assessed by a Public Accountant of Reputer verwendet. ${ }^{85}$

Bei der Veräußerung von Anteilen durch einen Vertragspartner wird zunächst ein 'Preexecutive Right ‘ des anderen Partner vereinbart. Dem Veräußerer obliegt eine Andienungspflicht, der eine Kaufoption des Partners korrespondiert. Nimmt letzterer dieses Erwerbsrecht nicht wahr, so unterliegt die Veräußerung an einen Dritten einem Zustimmungserfordernis des Vertragspartners. Die Zustimmung zum Verkauf darf allerdings nicht unreasonably verweigert werden.

\section{Geschäftsführung und Willensbildung}

Die gesamte Geschäftsführungsbefugnis - Rechtsverhältnis der Direktoren zu der Gesellschaft und zu ihren Aktionären - und die Vertretungsmacht - Rechtsverhältnis zu Dritten - liegt bei den Direktoren. Dieser 'Board of Directors' erfüllt die Funktion eines Vorstandes und eines Aufsichtsrates. Das indische Recht kennt, dem englischen Recht folgend, kein zweistufiges System: Vorstand - Aufsichtsrat, sondern folgt dem 'One Tier System $:^{86}$ Zwischen dem 'Board of Directors` und der Hauptversammlung ist kein weiteres Kontrollorgan eingeschaltet. Allerdings ergibt sich in der Regel eine Zweiteilung innerhalb des 'Boards'. Die laufende Geschäftsführung wird auf einzelne zum 'Board' gehörende 'Managing Directors` übertragen, während die übrigen 'Ordinary Directors` nur bei grundsätzlichen Entscheidungen mitwirken. Die Geschäftsführung und Vertretungsbefugnis des oder der 'Managing Director(s)، ist vergleichbar der eines Vorstands der Deutschen Aktiengesellschaft. Für den deutschen Partner ist die Rechtsfigur des 'Alternate Directors von Interesse. Ein Direktor verliert bei dreimonatiger Abwesenheit sein Amt. Wenn sich die Entsendung eines Direktors nach Indien in größeren Zeitabständen nicht lohnt, bietet die Bestellung eines 'Alternate Directors ‘ - meist ortsansässige Rechtsanwälte, Wirtschaftsprüfer oder Geschäftsleute - einen Ausweg. ${ }^{87}$ So wird die Anwesenheitspflicht erfüllt und die Einflußmöglichkeit gewahrt.

Für den deutschen Partner mit einer Minderheitsbeteiligung ergibt sich vor allem die Frage nach der Sicherung seines Einflusses. Die juristischen Möglichkeiten liegen in erster Linie bei der Besetzung der gemeinsamen Organe und Festlegung der erforderlichen Mehrheiten für bestimmte Geschäfte. ${ }^{88}$ So gestattet das indische Recht die Wahl der Direktoren durch Verhältniswahl. Inhaber beträchtlicher Minderheitsanteile können, wenn sie im Board of Directors vertreten sein wollen, dies durch eine Bestimmung in der Satzung sichern. Nach dem Companies Act werden die Entscheidungen des Board of Directors mit einfacher Mehrheit getroffen. In der Satzung kann allerdings ein Katalog wich-

85 Singhania, (Fn. 79), S. 4.

86 Vgl. Warekar, (Fn. 60), S. 18.

87 Vgl. Sevin, (Fn. 61), S. 33.

88 Dazu allgemein: Moecke, Vertragsgestaltung bei Zusammenarbeit auf dritten Märkten, in: RIW/AWD 1978, S. 73 (78). 
tiger Angelegenheiten aufgenommen werden, deren Entscheidung einer qualifizierten Dreiviertel- oder Zweidrittelmehrheit bedarf. Darüber hinaus kann eine Klausel vereinbart werden, wonach bedeutende Änderungen des Gesellschaftsvertrages, der Satzung sowie des Grundkapitals oder die Gewinnverteilung einschließlich der Dividendenausschüttung nicht ohne Zustimmung einer deutschen Sperrminorität ${ }^{89}$ beschlossen werden dürfen. Schließlich bedürfen in einer Public Company bestimmte Entscheidungen wie etwa die Ernennung von Direktoren, die Aufnahme von Krediten über den Beitrag des eingezahlten Kapitals und der freien Rücklagen hinaus oder die Verfügung über Teile des Unternehmens ohnehin der Zustimmung durch die Hauptversammlung. Bedeutende Entscheidungen können hier nur durch eine Dreiviertelmehrheit der Hauptversammlung gefällt werden.

\section{Anwendbares Recht und Schiedsgerichtsbarkeit}

Das auf den Vertrag des Gemeinschaftsunternehmens anwendbare Recht ist indisches Recht. Diese gleichsam erzwungene Rechtswahl ${ }^{90}$ des für deutsche Unternehmen ausländischen Rechts gehört schon zum Genehmigungserfordernis der indischen Regierung. Sie bietet sich insofern an, als nicht nur der indische Partner dort seinen Sitz hat, sondern auch der deutsche Partner seine wesentlichen Leistungen in Indien erbringt, und somit eine Verknüpfung beider Partner zum Standort Indien vorliegt. ${ }^{91}$

Vertragsstörungen versuchen die betroffenen Vertragspartner unter sich zu lösen. Unüblich ist die Bestellung eines unparteiischen Dritten, der dann ein Schiedsgutachten erstellen soll. Oft bewährt sich ein Hintereinanderschalten von anteilig besetzten 'Instanzen: Bereits die Tatsache, in einem nächsten Gremium antreten zu müssen, da in der vorherigen Instanz keine Lösung erzielt werden konnte, fördert die Bereitschaft, nach einer gemeinschaftlichen Kompromißlösung zu suchen.92

Eine ähnliche Wirkung haben schließlich die als ultima ratio eingesetzten Schiedsgerichtsverfahren. Schiedsgerichtklauseln, in denen die Zuständigkeit eines Schiedsgerichts außerhalb Indiens vereinbart wird, sind zulässig. Viele Gemeinschaftsunternehmen vereinbaren die Schiedsgerichtsklauseln der Internationalen Handelskammer in Paris und als Sitz des Schiedsgerichts London, Paris oder Zürich. Es kann sich also ein vom Vertragsstatut abweichendes Recht für das Schiedsverfahren ergeben. Indien hat das New-Yorker UN-Abkommen über die Anerkennung und Vollstreckung ausländischer Schiedsprüche vom 10. 6. 1958 unterzeichnet. Danach hat es ausländische Schiedssprüche so anzuerkennen und zu vollstrecken wie inländische Schiedssprüche. Es erließ nach

89 Singhania, (Fn. 79), S. 10.

90 Jayme, Komplexe Langzeitverträge und Internationales Privatrecht, in: IPrax 87, S. 63 (64).

91 Zweigert/v. Hofmann, (Fn. 5), S. 208; Ebenroth, (Fn. 80), a.a.O. will dies auch auf den Gründungsvorvertrag erstrecken, da am Sitz des Gemeinschaftsunternehmen die korrespondierenden wirtschaftlichen Interessen beider Partner rechtlich kulminieren.

92 Vgl. Schaub, (Fn. 81), S. 185. 
dem Beitritt zu diesem Ubereinkommen 1961 das 'Foreign Awards (Recognition and Enforcement) Act. Ublich ist es auch, sich der mit Praktikern besetzten Schiedsgerichtsbarkeit der Deutsch-Indischen oder Indischen Handelskammern zu bedienen. Für die Vollstreckung sowohl ausländischer wie inländischer Schiedssprüche ist eine Anerkennung indischer Gerichte erforderlich. Dieses Anerkennungsverfahren ist häufig langwierig, da die vor dem Schiedsgericht unterlegene Partei gegen die gerichtliche Anerkennung Formfehler des vorangegangenen Schiedsverfahrens geltend macht. 


\title{
Indo-German Joint Ventures in India
}

\author{
By Tillmann R. Braun
}

Joint ventures offer an established compromise between the political interests of the developing countries in sufficient control and the economic goals of the industrial nations' enterprises in exploring the local market. The joint ventures are to realize through their new and self-supporting legal entities a financial, technical and managerial cooperation within certain contractual conditions between Indian and German partners. The latter are mainly interested in serving the Indian market while the essential motive for the Indian partners is the acquisition and utilization of advanced technology. The political and economic rationale for the ambivalent attitude of the Indian Government towards foreign direct investment is described; it has led in the past to a somewhat variable and changing legislation on foreign investment which in reality has subjected the foreign investor to certain limitation in his possibilities and activities.

Important bilateral international traties, the Capital Protection Agreement and Double Taxation Agreement, are reviewed as they set binding international standards for the national investment legislation in these areas. The Indian investment legislation and its respective instruments are limiting the foreign shareholding in enterprises in India principally to $40 \%$ in order to avoid salienation'; few special exceptions are granted on merits to high-technology and export-orientated industries: Foreign investors are excluded totally from banking, trade and insurance as well as from the production of consumer goods. Finally, particular provisions of the Indian Company Law and contractual clauses of Indo-German Joint Ventures are examined.

\section{Intergovernmental Commodity Regimes in a Twilight - Lessons from the Tin Debacle}

\section{By Ludwig Gramlich}

The collapse of the Sixth International Tin Agreement (ITC) in the autumn of 1985 came somewhat surprisingly, given the rather successful history of the regulation of international commodity trade in the non-agricultural field. Where are the origins and causes of the ITC debacle? Are they typical only for his special kind of existing type(s) of Intergovernmental Commodity Agreements (ICAs)? The author tries to give some answers to these important questions, dealing at first with two regulatory patterns of international commodity trade after 1945, the Havana Charter model and the more recent one, related to the activities of UNCTAD and to the ideas of a new international economic order. Then, the national economies of certain essential tin-producing countries as well as the different legal regimes for mining within those states and the relationship between international and internal factors are described in more detail. The last two sec- 